\title{
Length-scale estimates for the LANS- $\alpha$ equations in terms of the Reynolds number
}

\author{
J.D. Gibbon*, D.D. Holm \\ Department of Mathematics, Imperial College London, London SW7 2AZ, UK
}

Received 28 April 2006; received in revised form 27 June 2006; accepted 28 June 2006

Communicated by U. Frisch

\begin{abstract}
Foias, Holm and Titi [C. Foias, D.D. Holm, E.S. Titi, The three dimensional viscous Camassa-Holm equations and their relation to the Navier-Stokes equations and turbulence theory, J. Dynam. Differential Equations 14 (2002) 1-35] have settled the problem of existence and uniqueness for the 3D LANS- $\alpha$ equations on periodic box $[0, L]^{3}$. There still remains the problem, first introduced by Doering and Foias [C.R. Doering, C. Foias, Energy dissipation in body-forced turbulence, J. Fluid Mech. 467 (2002) 289-306] for the Navier-Stokes equations, of obtaining estimates in terms of the Reynolds number $R e$, whose character depends on the fluid response, as opposed to the Grashof number, whose character depends on the forcing. $R e$ is defined as $R e=U \ell / v$ where $U$ is a bounded spatio-temporally averaged Navier-Stokes velocity field and $\ell$ the characteristic scale of the forcing. It is found that the inverse Kolmogorov length is estimated by $\ell \lambda_{k}^{-1} \leq c(\ell / \alpha)^{1 / 4} R e^{5 / 8}$. Moreover, the estimate of Foias, Holm and Titi for the fractal dimension of the global attractor, in terms of $R e$, comes out to be
\end{abstract}

$$
d_{F}(\mathcal{A}) \leq c \frac{V_{\alpha} V_{\ell}^{1 / 2}}{\left(L^{2} \lambda_{1}\right)^{9 / 8}} R e^{9 / 4}
$$

where $V_{\alpha}=\left(L /(\ell \alpha)^{1 / 2}\right)^{3}$ and $V_{\ell}=(L / \ell)^{3}$. It is also shown that there exists a series of time-averaged inverse squared length scales whose members, $\left\langle\kappa_{n, 0}^{2}\right\rangle$, are estimated as $(n \geq 1)$

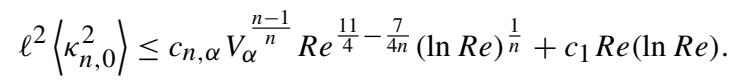

The upper bound on the first member of the hierarchy $\left\langle\kappa_{1,0}^{2}\right\rangle$ coincides with the inverse squared Taylor micro-scale to within log-corrections. (C) 2006 Elsevier B.V. All rights reserved.

Keywords: $\alpha$-model; Navier-Stokes; Turbulence; Lagrangian-averaged; Existence; Uniqueness; Camassa-Holm; Length-scale; Global attractor; Fractal dimension

\section{Introduction}

\subsection{Background to the LANS- $\alpha$ model}

Turbulence is a state of continual unrest, which arises as a fluid's response to forcing by stirring, for example, or by flowing along a wall. The scaling properties of turbulence are characterized by two dimensionless numbers. These are

\footnotetext{
* Corresponding author. Tel.: +44 2075948504.

E-mail address: j.d.gibbon@ic.ac.uk (J.D. Gibbon).
}

the Grashof number $G r$, which measures forcing, and the Reynolds number $R e$ which characterizes the fluid's response to the forcing. The turbulent response to forcing produces strong fluctuations in the fluid motion whose statistics obey power law spectra extending over a large range of length scales and timescales [1,2]. This fluctuating multi-scale response is the hallmark of turbulence. Turbulence researchers often characterize the development of the multi-scale response as a "cascade" of kinetic energy rushing downward from the larger fluid motions due to forcing to the smaller and smaller circulations of eddies, sheets, and tubes of vorticity. In 
stretching themselves into finer and finer shapes, these vortical structures comprise the "sinews" of turbulence.

Characteristic features of turbulence - its distribution of eddy sizes, shapes, speeds, vorticity, circulation and viscous dissipation - may all be captured by using the exact Navier-Stokes equations. These correctly predict how the cascade of turbulent kinetic energy and vorticity accelerates and how the sinews of turbulence stretch themselves into finer and finer scales, until their motions reach only a few molecular mean free paths, where they may finally be dissipated by viscosity into heat: for more details, see [2-7]. However, the fidelity of the Navier-Stokes equations in capturing the cascade of turbulence is also the cause of serious problems in direct numerical simulations of turbulence.

Based on Landau's heuristic idea that the number of active degrees of freedom required to simulate the turbulent cascade in high-Reynolds-number flows increases as $R e^{9 / 4}$, it is clear that this geometric rate of increase quickly outstrips the numerical resolution capabilities of even the largest computer. To make turbulence computable, scientists have developed various approximate models that halt the cascade into smaller, faster eddies. In most models, this effect is accomplished by causing the eddies below a certain size to dissipate computationally into heat. This dissipative imperative causes errors, however, because it damps out the variability (known as intermittency) in the larger-scale flow, which is caused by the myriad of small scales of motion interacting nonlinearly together in the fields of the larger motions. Thus, computational turbulence closure models based on enhancing viscous dissipation over its physical Navier-Stokes value run the risk of producing unrealistically low variability.

Perhaps surprisingly, one of the first clues in understanding how to develop turbulence closure models without enhancing viscous dissipation came from the great mathematical analyst Leray [8] who showed how to regularize the Navier-Stokes equations by modifying their nonlinearity to the well-known form

$\boldsymbol{v}_{t}+\boldsymbol{u} \cdot \nabla \boldsymbol{v}+\nabla p=v \Delta \boldsymbol{v}+\boldsymbol{f}(\boldsymbol{x}), \quad \operatorname{div} \boldsymbol{v}=0$,

with $\boldsymbol{v}=0$ on the boundary. Here $v$ is the (constant) kinematic viscosity coefficient, $\boldsymbol{f}(\boldsymbol{x})$ is the prescribed external force and $\boldsymbol{u}=G_{\delta} * \boldsymbol{v}$ is a filtered version of the regularized velocity $\boldsymbol{v}$. The filtering operation is defined by $G_{\delta} * \boldsymbol{v}=\int G_{\delta}(\boldsymbol{x}, \mathbf{y}) \boldsymbol{v}(\mathbf{y}) \mathrm{d}^{3} y$ for a symmetric kernel $G_{\delta}(\boldsymbol{x}, \mathbf{y})$ of characteristic width $\delta$. The Navier-Stokes equations for $\boldsymbol{v}$ are recovered in the limit as $\delta \rightarrow 0$, so that $\boldsymbol{u} \rightarrow \boldsymbol{v}$. For a review of the Leray regularization of the Navier-Stokes equations, see Gallavotti [9].

One of the points made in [9] is that the Leray regularization of the Navier-Stokes equations no longer satisfies the Kelvin circulation theorem, since for these equations

$$
\begin{aligned}
\frac{\mathrm{d}}{\mathrm{d} t} \oint_{\Gamma(\boldsymbol{u})} \boldsymbol{v} \cdot \mathrm{d} \boldsymbol{x} & =\oint_{\Gamma(\boldsymbol{u})}\left(\boldsymbol{v}_{t}+\boldsymbol{u} \cdot \nabla \boldsymbol{v}+\nabla \boldsymbol{u}^{\mathrm{T}} \cdot \boldsymbol{v}\right) \cdot \mathrm{d} \boldsymbol{x} \\
& \neq \oint_{\Gamma(\boldsymbol{u})}(v \Delta \boldsymbol{v}+\boldsymbol{f}) \cdot \mathrm{d} \boldsymbol{x} .
\end{aligned}
$$

Remarkably, combining the process of Lagrangian averaging with Taylor's hypothesis (that fluctuations have such low power that they may be regarded as being carried along by the mean flow) leads to a regularized set of equations which answers the challenge of [9] to produce a regularization of the Navier-Stokes equations which do possess a Kelvin circulation theorem. These regularized equations comprise the LANS$\alpha$ model $^{1}$

$\boldsymbol{v}_{t}+\boldsymbol{u} \cdot \nabla \boldsymbol{v}+\nabla \boldsymbol{u}^{\mathrm{T}} \cdot \boldsymbol{v}+\nabla P=v \Delta \boldsymbol{v}+\boldsymbol{f}(\boldsymbol{x}), \quad \operatorname{div} \boldsymbol{u}=0$,

where $P=p-\frac{1}{2} \nabla\left(|\boldsymbol{u}|^{2}+\alpha^{2}|\nabla \boldsymbol{u}|^{2}\right)$. An equivalent alternative formulation is to rewrite (1.3) as

$\boldsymbol{v}_{t}-\boldsymbol{u} \times \operatorname{curl} \boldsymbol{v}=v \Delta \boldsymbol{v}-\nabla \tilde{p}+\boldsymbol{f}(\boldsymbol{x})$

where $\tilde{p}=P+\boldsymbol{u} \cdot \boldsymbol{v}$. Usually, $\alpha$ is taken as a constant with dimension of length and the filtering relation $\boldsymbol{u}=G_{\alpha} * \boldsymbol{v}$ for the advection velocity in the LANS- $\alpha$ model is specified as

$\boldsymbol{v} \equiv \boldsymbol{u}-\alpha^{2} \Delta \boldsymbol{u}$

Some remarks are in order here:

1. The filtering kernel $G_{\alpha}$ for the LANS- $\alpha$ model thus turns out to be the Green's function for the Helmholtz operator $\left(1-\alpha^{2} \Delta\right)$.

2. As expected, the LANS- $\alpha$ motion equation satisfies the Kelvin circulation theorem:

$$
\begin{aligned}
\frac{\mathrm{d}}{\mathrm{d} t} \oint_{\Gamma(\boldsymbol{u})} \boldsymbol{v} \cdot \mathrm{d} \boldsymbol{x} & =\oint_{\Gamma(\boldsymbol{u})}\left(\boldsymbol{v}_{t}+\boldsymbol{u} \cdot \nabla \boldsymbol{v}+\nabla \boldsymbol{u}^{\mathrm{T}} \cdot \boldsymbol{v}\right) \cdot \mathrm{d} \boldsymbol{x} \\
& =\oint_{\Gamma(\boldsymbol{u})}(v \Delta \boldsymbol{v}+\boldsymbol{f}) \cdot \mathrm{d} \boldsymbol{x}
\end{aligned}
$$

The circulation theorem tells us that the rate of change of circulation of momentum per unit mass $\boldsymbol{v}$ around a closed material loop $\Gamma(\boldsymbol{u})$ moving with velocity $\boldsymbol{u}=G_{\alpha} * \boldsymbol{v}$ is due to the integral around that loop of the tangential component of the sum over forces (viscous and external) acting on the fluid.

3. This statement of the circulation theorem is also a mnemonic for deriving other regularized turbulence models of LANS- $\alpha$ type by specifying a different filtering kernel $G_{\alpha}[10]$.

We have seen that the LANS- $\alpha$ model can be immediately derived from its circulation theorem. However, the approach used historically in Chen et al. [11] and Holm and Titi [12] for deriving the closed Eulerian form (1.3) of the LANS- $\alpha$ motion equation was based on the combination of two other earlier results. First, the Lagrangian-averaged variational principle of Gjaja and Holm [13] was applied for deriving the inviscid averaged nonlinear fluid equations, obtained by averaging Hamilton's principle for fluids over the rapid phase of their small turbulent circulations at fixed Lagrangian coordinate: this

\footnotetext{
${ }^{1}$ LANS is an acronym standing for "Lagrangian-averaged Navier-Stokes" while alpha $(\alpha)$ is the coherence length of the Lagrangian statistics; this term will be used hereafter.
} 
step had its own precedent in earlier work on Lagrangianaveraged fluid equations by Andrews and McIntyre [14]. Second, the Euler-Poincaré theory for continuum mechanics of Holm et al. [15] was used for handling the Eulerian form of the resulting Lagrangian-averaged fluid variational principle. This second step determined the relationships among the momentum per unit mass $\boldsymbol{v}$, the Lagrangian-averaged velocity of the fluid $\boldsymbol{u}$ and the Lagrangian fluctuation statistics. Next, Taylor's hypothesis of frozen-in turbulence circulations was invoked for closing the Eulerian system of Lagrangian-averaged fluid equations, by obtaining the explicit relation $\boldsymbol{v} \equiv \boldsymbol{u}-\alpha^{2} \Delta \boldsymbol{u}$. Finally, Navier-Stokes Eulerian viscous dissipation was added, so that viscosity would cause diffusion of the newly defined Lagrangian-average momentum and monotonic decrease of its total Lagrangian-averaged energy.

At this point, one may regard the LANS- $\alpha$ model as an alternative regularization of the Navier-Stokes equations and re-examine its properties from the viewpoint of Leray's analysis: in fact the problem of existence and uniqueness for the LANS- $\alpha$ equations has already been settled by Foias et al. [16]. In addition, the same ideas which restore Kelvin's circulation theorem to Leray's regularization of the Navier-Stokes equations also turn out to provide a basis for deriving candidate equations for Large Eddy Simulations (LES) of turbulence. Conversely, other proposed LES models of turbulence may lead to likely candidates for application of Leray's analysis. In this way, the classical Leray analysis of the Navier-Stokes equations finds itself a new role in the study of the analytical properties of turbulence models. Indeed, the Leray model itself was recently found to be a viable candidate for LES computational modeling of turbulence [10,17].

\subsection{Estimates in terms of Reynolds number}

Motivated by the Navier-Stokes equations, in an early and progressive paper Ruelle [18] discussed how ideas in dynamical systems might be extended to the infinite dimensional case by counting the number of positive characteristic exponents. Certain general finiteness assumptions were made about the nature of the Navier-Stokes equations without formally using the concept of a global attractor. These ideas were taken to another level by Constantin and Foias [19], who used the idea of a global attractor $\mathcal{A}$ for a partial differential equation to determine the number of growing Lyapunov exponents and thence, through a rigorous generalization of the Kaplan-Yorke formula, they were able to get formal upper bounds on $d_{L}(\mathcal{A})$, the Lyapunov dimension of $\mathcal{A}$, which itself bounds above $d_{F}(\mathcal{A})$ and $d_{H}(\mathcal{A})$. Importantly these bounds depend only upon time-averages and thus only on the long-time dynamics on $\mathcal{A}$; see also [20]. When applied to the two-dimensional Navier-Stokes equations, for which a global attractor exists, these methods gave good, sharp estimates to within logarithmic corrections [21] when used in conjunction with an $L^{\infty}$ estimate of Constantin [22]. The development of the key features of these ideas can be found in $[3,23]$, including the role played by the inequalities of Lieb and Thirring [24]. Apart from the twodimensional Navier-Stokes equations, several important partial differential equations possess a global attractor, such as the twodimensional complex Ginzburg-Landau equation and the onedimensional Kuramoto-Sivashinky equation. Unfortunately, the three-dimensional Navier-Stokes equations are not among them; the key element is the lack of a proof of existence and uniqueness without which the existence of a global attractor remains open.

In settling the question of existence and uniqueness for the LANS- $\alpha$ equations, Foias et al. [16] were able to use the machinery developed for global attractors to find estimates for the dimension of its global attractor $\mathcal{A}$ (and other important quantities) in terms of the Grashof number $G r$, which is a dimensionless control parameter dependent only on the ratio of the forcing to the viscosity $v$. Therefore, as a regularization of the three-dimensional Navier-Stokes equations with many similar features to its parent, the LANS- $\alpha$ equations possess this extra key property. What remains to be proved, however, is whether the estimates in [16] can be evaluated in terms of the Reynolds number, whose character depends on the fluid response to the forcing, and which is intrinsically a property of Navier-Stokes solutions. The advantage of this further step lies in the fact that the engineering and physics communities express their ideas about turbulence in terms of the Reynolds number. It also allows us to make direct comparisons between estimates for the two equations where they exist (see Table 1).

For simplicity the LANS- $\alpha$ equations (1.3), or their alternative form in (1.4), will be considered on a periodic domain $[0, L]_{\text {per }}^{3}$ with forcing $\boldsymbol{f}(\boldsymbol{x})$ taken to be $L^{2}$-bounded of narrow-band type ${ }^{2}$ with a single length-scale $\ell$ (see [30-32])

$\left\|\nabla^{n} \boldsymbol{f}\right\|_{2} \approx \ell^{-n}\|\boldsymbol{f}\|_{2}$.

With $f_{\text {rms }}=L^{-d / 2}\|\boldsymbol{f}\|_{2}$, where $\|\boldsymbol{f}\|_{2}^{2}=\int_{\Omega}|\boldsymbol{f}|^{2} \mathrm{~d} V$, the standard definition of the Grashof number in $d$ dimensions is

$G r=\frac{\ell^{3} f_{\mathrm{rms}}}{v^{2}}$.

Analytical estimates in Navier-Stokes theory have traditionally been expressed in terms of $G r$ [3,4,25-29] but these are difficult to compare with the results of Kolmogorov scaling theories which are expressed in terms of Reynolds number [2]. A good definition of this is

$R e=\frac{U \ell}{v} \quad U^{2}=L^{-d}\left\langle\|\boldsymbol{u}\|_{2}^{2}\right\rangle$

where $\langle\cdot\rangle$ is the long-time average

$$
\langle g(\cdot)\rangle=\varlimsup_{t \rightarrow \infty} \frac{1}{t} \int_{0}^{t} g(\tau) \mathrm{d} \tau .
$$

Doering and Foias [30] addressed this problem recently and have shown that in the limit $G r \rightarrow \infty$, solutions of the $d$ dimensional Navier-Stokes equations

$\boldsymbol{u}_{t}+\boldsymbol{u} \cdot \nabla \boldsymbol{u}+\nabla p=v \Delta \boldsymbol{u}+\boldsymbol{f}(\boldsymbol{x}), \quad \operatorname{div} \boldsymbol{v}=0$

\footnotetext{
2 The restriction to narrow-band forcing can be relaxed at the cost of more parameters in the problem.
} 
Table 1

Comparison of various time-average bounds for the Navier-Stokes and LANS- $\alpha$ equations with constants omitted

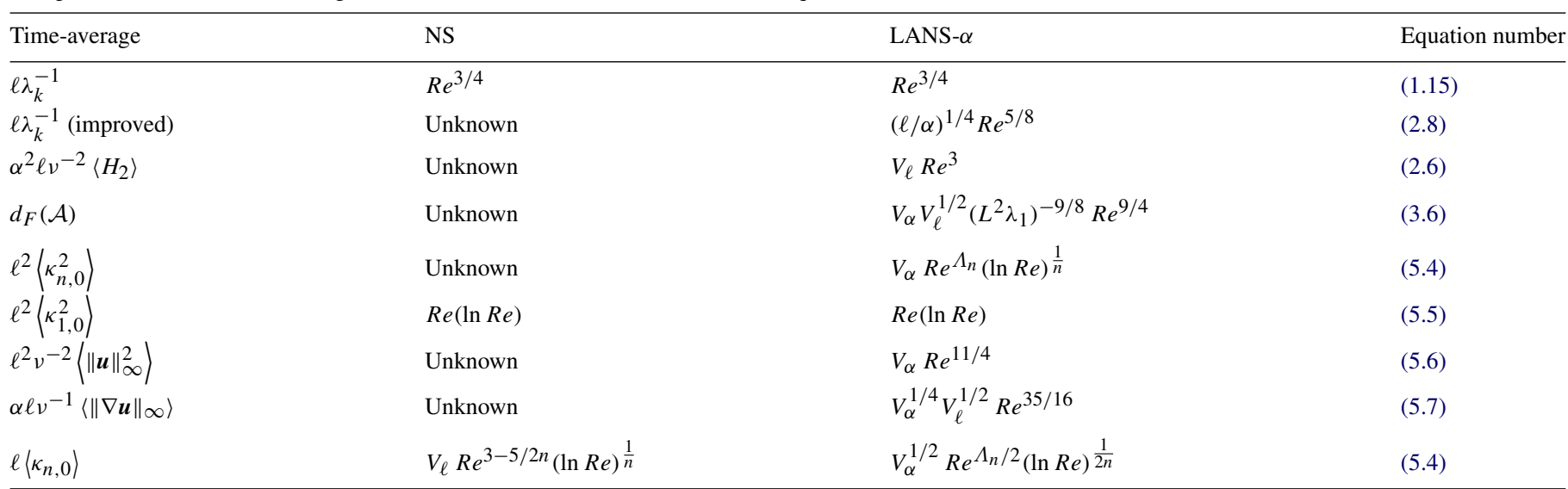

Note that in the cases where there is no known equivalent upper bound for the Navier-Stokes equations, the LANS- $\alpha$ upper bounds blow up as $\alpha \rightarrow 0: \Lambda_{n}$ is defined in (1.23). Lines 5-8 are a summary of the results of Theorem 2 in Section 5. The Navier-Stokes estimate for $\ell\left\langle\kappa_{n, 0}\right\rangle$ on the last line is there for comparative purposes and is taken from [31,32].

must satisfy ${ }^{3}$

$G r \leq c\left(R e^{2}+R e\right)$

Using the relation in (1.12), Doering and Gibbon [31,32] have re-expressed some 3D Navier-Stokes estimates in terms of $R e$ (see Table 1). The problem, however, is less simple than substituting (1.12) into standard results, although this works well enough for pointwise estimates [16]. Time averages are more subtle and exploit how the average velocity $U$ within $R e$ is related to $\left\langle\|\boldsymbol{u}\|_{2}^{2}\right\rangle$. As an illustration, let us consider the Navier-Stokes equation (1.11) whose energy dissipation rate is $\epsilon=v\left\langle\|\nabla \boldsymbol{u}\|_{2}^{2}\right\rangle L^{-d}$. Standard estimates show that its upper bound is proportional to $G r^{2}$. By (1.12), this turns into $R e^{4}$, which is not sharp. Now we estimate this a different way [30]: consider Leray's energy inequality

$\frac{1}{2} \frac{\mathrm{d}}{\mathrm{d} t}\|\boldsymbol{u}\|_{2}^{2} \leq-v\|\nabla \boldsymbol{u}\|_{2}^{2}+\|\boldsymbol{f}\|_{2}\|\boldsymbol{u}\|_{2}$.

Time-averaging (1.13) and using (1.9) and (1.12) yields

$\epsilon \leq v^{3} \ell^{-4} G r \operatorname{Re} \leq c v^{3} \ell^{-4}\left(R e^{3}+R e\right)$,

which is a considerable improvement. To leading order the inverse Kolmogorov length $\lambda_{k}^{-1}=\left(\epsilon / v^{3}\right)^{1 / 4}$ is then bounded above by

$\ell \lambda_{k}^{-1} \leq c R e^{3 / 4}$.

This estimate now conforms with the generally accepted scaling law for the inverse Kolmogorov length with the Reynolds number $[1,2]$.

The relation in (1.12) is essentially a Navier-Stokes result and thus needs re-proving for the LANS- $\alpha$ equations. It turns out to be true but the proof is a non-trivial extension of the

\footnotetext{
3 This result is not advertised in [30] but it follows immediately from their Eq. 48. Ref. [30] also contains another result that the energy dissipation rate $\epsilon$ has a lower bound proportional to $G r$ : see Appendix A.2.
}

method in [30]; the whole of Appendix A.1 is devoted to this proof. As will be shown in Section 2, the estimate for $\left\langle H_{1}\right\rangle$ in (1.14) can be improved to

$\epsilon \leq c v^{3} \ell^{-3} \alpha^{-1} R e^{5 / 2}, \quad \Rightarrow \ell \lambda_{k}^{-1} \leq c\left(\frac{\ell}{\alpha}\right)^{1 / 4} R e^{5 / 8}$

Moreover, in Section 3 the estimate by Foias et al. [16] (see also [33]) for the fractal dimension $d_{F}(\mathcal{A})$ of the global attractor $\mathcal{A}$ is considered in the light of these $R e$-bounds. Using their estimate in terms of the generalized $\alpha$-dependent dissipation rate, we show that

$d_{F}(\mathcal{A}) \leq c \frac{V_{\alpha} V_{\ell}^{1 / 2}}{\left(L^{2} \lambda_{1}\right)^{9 / 8}} R e^{9 / 4}$

where the two dimensionless volumes $V_{\ell}$ and $V_{\alpha}$ are defined by

$V_{\ell}=\left(\frac{L}{\ell}\right)^{3} \quad V_{\alpha}=\left(\frac{L}{(\ell \alpha)^{1 / 2}}\right)^{3}$

and $\lambda_{1}>0$ is the smallest eigenvalue of the Stokes operator. Given our definition of $R e$ in (1.9), this $R e^{9 / 4}$ estimate is consistent with scaling theories of turbulence but it does not survive in the Navier-Stokes limit because the volume $V_{\alpha}$ blows up as $\alpha \rightarrow 0$.

The $R e^{9 / 4}$ estimate for $d_{F}$ also gives an idea of how many degrees of freedom, in Landau's sense, exist in a turbulent flow-indeed this is exactly the result predicted by Landau for the Navier-Stokes equations. ${ }^{4}$ What is not taken into account in this picture is the effect of strong dissipation-range intermittency where significant energy lies in wave-numbers larger than $\lambda_{k}^{-1}$. In this case estimates are needed for lengthscales that are associated with higher derivatives. This idea has been investigated in $[31,32]$ where estimates were found for

\footnotetext{
${ }^{4}$ However, given the improved $R e^{5 / 8}$ inverse Kolmogorov estimate in (1.16) for the LANS- $\alpha$ model, it is possible that the sharp estimate for $d_{F}$ is proportional to $R e^{15 / 8}$.
} 
time-averaged Navier-Stokes quantities. Those for the LANS$\alpha$ equations should be much better because of their enhanced regularity properties [16]. In Section 4 we combine the forcing with higher derivatives of the velocity field in the form

$F_{n}=H_{n}+\tau^{2}\left\|\nabla^{n} \boldsymbol{f}\right\|_{2}^{2}$,

where $\tau=\ell^{2} v^{-1}(G r \ln G r)^{-1 / 2}$ is a characteristic time: see Appendix A.2. We also form the combination

$J_{n}=F_{n}+\alpha^{2} F_{n+1}$

and use it to define a set of inverse length scales, or timedependent wave-numbers,

$\kappa_{n, 0}(t)=\left(\frac{J_{n}}{J_{0}}\right)^{\frac{1}{2 n}}$.

In the $\alpha \rightarrow 0$ limit, the $\kappa_{n, 0}^{2 n}$ behave as the $2 n$th moments of the energy spectrum. Theorem 2 of Section 4 proves that the time average of their squares must obey

$\ell^{2}\left\langle\kappa_{n, 0}^{2}\right\rangle \leq c_{n, \alpha} V_{\alpha}^{\frac{n-1}{n}} R e^{\Lambda_{n}}(\ln R e)^{\frac{1}{n}}+c_{1} R e \ln R e$,

for the LANS- $\alpha$ model, where

$\Lambda_{n}=\frac{11}{4}-\frac{7}{4 n}$.

Note that the $n=1$ estimate in (1.22) scales with $R e$ the same as the Taylor micro-scale. This best that could be achieved for the full 3D Navier-Stokes equations was [31,32]

$\ell\left\langle\kappa_{n, 0}\right\rangle \leq c_{n} V_{\ell} R e^{3-\frac{5}{2 n}}(\ln R e)^{\frac{1}{n}}+c_{1} R e \ln R e$,

which appears in the last line of Table 1. With the exponent of unity in the time-average - if indeed a solution exists at all this is not only much worse that (1.22) but represents only weak solutions, as opposed to the strong solutions of Foias et al. [16]. The fact that no upper bound is known to exist for $\left\langle\kappa_{n, 0}^{2}\right\rangle$ for the 3D Navier-Stokes equations is consistent with the fact that the dimensionless volume $V_{\alpha}$ blows up as $\alpha \rightarrow 0$. The $\kappa_{n, 0}$ could even become singular in this limit.

\section{Properties of the product $\int_{\Omega} u \cdot v \mathrm{~d} V$}

Foias et al. [16] noted that the product $\boldsymbol{u} \cdot \boldsymbol{v}$ has two convenient properties

$$
\int_{\Omega} \boldsymbol{u} \cdot \boldsymbol{v} \mathrm{d} V=\int_{\Omega}\left\{|\boldsymbol{u}|^{2}+\alpha^{2}|\nabla \boldsymbol{u}|^{2}\right\} \mathrm{d} V
$$

and

$$
\begin{aligned}
\frac{\mathrm{d}}{\mathrm{d} t} \int_{\Omega} \boldsymbol{u} \cdot \boldsymbol{v} \mathrm{d} V & =\int_{\Omega}\left(\boldsymbol{u}_{t} \cdot \boldsymbol{v}+\boldsymbol{u} \cdot \boldsymbol{v}_{t}\right) \mathrm{d} V \\
& =\int_{\Omega}\left(\boldsymbol{u}_{t} \cdot\left(1-\alpha^{2} \Delta\right) \boldsymbol{u}+\boldsymbol{u} \cdot \boldsymbol{v}_{t}\right) \mathrm{d} V \\
& =\int_{\Omega}\left\{\boldsymbol{u} \cdot\left[\left(1-\alpha^{2} \Delta\right) \boldsymbol{u}_{t}\right]+\boldsymbol{u} \cdot \boldsymbol{v}_{t}\right\} \mathrm{d} V \\
& =2 \int_{\Omega} \boldsymbol{u} \cdot \boldsymbol{v}_{t} \mathrm{~d} V
\end{aligned}
$$

where two integrations by parts have occurred between the second and third lines. Now define

$H_{n}=\int_{\Omega}\left|\nabla^{n} \boldsymbol{u}\right|^{2} \mathrm{~d} V \equiv \int_{\Omega}\left|\operatorname{curl}^{n} \boldsymbol{u}\right|^{2} \mathrm{~d} V$

this being true on a periodic domain because div $\boldsymbol{u}=0$. Clearly we have the bound

$$
\begin{aligned}
\frac{1}{2} \frac{\mathrm{d}}{\mathrm{d} t}\left(H_{0}+\alpha^{2} H_{1}\right) & =-v\left(H_{1}+\alpha^{2} H_{2}\right)+\int_{\Omega} \boldsymbol{u} \cdot \boldsymbol{f} \mathrm{d} V \\
& \leq-v\left(H_{1}+\alpha^{2} H_{2}\right)+\|\boldsymbol{u}\|_{2}\|\boldsymbol{f}\|_{2} .
\end{aligned}
$$

One can then calculate an absorbing ball for $H_{1}$ with ease (see [16]). It is also possible to estimate the time averages $\left\langle H_{1}\right\rangle$ and $\left\langle H_{2}\right\rangle$ which can be found in the same manner as in (1.13) to satisfy

$v L^{-3}\left\langle H_{1}+\alpha^{2} H_{2}\right\rangle \leq v^{3} \ell^{-4} \operatorname{Re} G r \leq c v^{3} \ell^{-4} \operatorname{Re}^{3}$.

The upper bound on $\left\langle H_{2}\right\rangle$, written as

$\alpha^{2} \ell v^{-2}\left\langle H_{2}\right\rangle \leq c V_{\ell} R e^{3}$

can then be used to improve the estimate for $\left\langle H_{1}\right\rangle$ by using the fact that $\left\langle H_{1}\right\rangle \leq\left\langle H_{0}\right\rangle^{1 / 2}\left\langle H_{2}\right\rangle^{1 / 2}$ and that $U^{2}=L^{-3}\left\langle H_{0}\right\rangle$. We find that

$\left\langle H_{1}\right\rangle \leq c v^{2} L^{3} \ell^{-3} \alpha^{-1} R e^{5 / 2}$

and so

$\ell \lambda_{k}^{-1} \leq c\left(\frac{\ell}{\alpha}\right)^{1 / 4} R e^{5 / 8}$.

Hence the energy dissipation rate $\epsilon$ is also bounded above by $R e^{5 / 2}$ but the improved estimate blows up when $\alpha \rightarrow 0$; no equivalent result is implied for the $3 \mathrm{D}$ Navier-Stokes equations.

\section{A $R e^{9 / 4}$ bound for the attractor dimension}

Foias et al. [16] made two independent estimates of the fractal dimension $d_{F}(\mathcal{A})$ of the global attractor $\mathcal{A}$. The first was in terms of $G r$ but the second estimate was made in terms of the "energy dissipation rate" $\bar{\epsilon}$; this phrase has been put in inverted commas because it includes the $\mathrm{H}_{2}$-norm, whereas conventionally only the $H_{1}$-norm is used. Their definition of $\bar{\epsilon}$ is

$\bar{\epsilon}=\lambda_{1}^{3 / 2} v\left\langle H_{1}+\alpha^{2} H_{2}\right\rangle$

where $\lambda_{1}$ is the smallest eigenvalue of the Stokes' operator which has the dimension of an inverse length squared. Foias et al. [16] then proved that

$d_{F}(\mathcal{A}) \leq c \frac{\lambda_{1}^{-3 / 2}}{\left(\alpha^{2} \lambda_{1}\right)^{3 / 4}}\left(\frac{\bar{\epsilon}}{v^{3}}\right)^{3 / 4}$.

They then defined a Kolmogorov length as $\ell_{\epsilon}^{-1}=\left(\bar{\epsilon} / \nu^{3}\right)^{1 / 4}$ which turns (3.2) into

$d_{F}(\mathcal{A}) \leq c \frac{1}{\left(\alpha^{2} \lambda_{1}\right)^{3 / 4}} \frac{1}{\left(\ell_{\epsilon} \lambda_{1}^{1 / 2}\right)^{3}}$. 
The problem here lies in interpretation: $\ell_{\epsilon}$ is not the conventional Kolmogorov length because $\bar{\epsilon}$ is not the Navier-Stokes energy dissipation rate $\epsilon=v\left\langle H_{1}\right\rangle L^{-3}$. Instead we take an alternative route and use the estimate for $\left\langle H_{1}+\alpha^{2} H_{2}\right\rangle$ from (2.5), which we repeat here

$v L^{-3}\left\langle H_{1}+\alpha^{2} H_{2}\right\rangle \leq c v^{3} \ell^{-4} R e^{3}$.

Thus

$\bar{\epsilon} \leq c\left(L \lambda_{1}^{1 / 2}\right)^{3} v^{3} \ell^{-4} R e^{3}$,

which turns the result of Foias et al. [16] into

$d_{F}(\mathcal{A}) \leq c \frac{V_{\alpha} V_{\ell}^{1 / 2}}{\left(L^{2} \lambda_{1}\right)^{9 / 8}} R e^{9 / 4}$,

where $L^{2} \lambda_{1}=4 \pi^{2}$. The right hand side blows up as $\alpha \rightarrow 0$ through $V_{\alpha}$. Despite this, the $R e^{9 / 4}$ estimate is, to our belief, the first time this has been achieved with this definition of $R e$. As has often been pointed out, this upper bound is also valid for the Hausdorff dimension $d_{H}(\mathcal{A})$ because $d_{H}(\mathcal{A}) \leq d_{F}(\mathcal{A})$.

\section{A theorem involving higher derivatives}

In terms of the number of degrees of freedom, the result in (3.6) says that $R e^{3 / 4} \times R e^{3 / 4} \times R e^{3 / 4}$ resolution grid points are needed. However, as explained in Section 1.2, what is not taken into account in attractor dimension estimates is the effect of strong dissipation-range intermittency where significant energy lies in wave-numbers larger than $\lambda_{k}^{-1}$. In this case estimates are needed for length-scales that are associated with higher derivatives. To obtain such estimates we begin by forming the combination

$F_{n}=H_{n}+\tau^{2}\left\|\nabla^{n} \boldsymbol{f}\right\|_{2}^{2}$

where the quantity $\tau$

$\tau=\ell^{2} v^{-1}(G r \ln G r)^{-1 / 2}$

where the $\ln G r$-term is there for reasons explained in Appendix A.2. We also define the combination

$J_{n}=F_{n}+\alpha^{2} F_{n+1}$.

The ultimate aim is to find time-averaged estimates for the $\kappa_{n, r}$ that appeared in (1.21): this will be the subject of the next section. In preparation, we prove the following result:

Theorem 1. As $G r \rightarrow \infty$, for $n \geq 1,1 \leq p \leq n$, $J_{n}$ satisfies

$$
\begin{aligned}
\frac{\mathrm{d} J_{n}}{\mathrm{~d} t} \leq & -\frac{1}{2} v \frac{J_{n}^{1+\frac{1}{p}}}{J_{n-p}^{1 / p}} \\
& +c_{n, \alpha} v^{-1}\|\boldsymbol{u}\|_{\infty}^{2} J_{n}+c_{1} v \ell^{-2} \operatorname{Re}(\ln R e) J_{n}
\end{aligned}
$$

and, for $n=0$,

$\frac{1}{2} \frac{\mathrm{d} J_{0}}{\mathrm{~d} t} \leq-v J_{1}+c_{1} v \ell^{-2} R e(\ln R e) J_{0}$.
Proof. The results on the pairing of $H_{0}$ and $H_{1}$ in (2.1) and (2.2) apply more generally:

$$
\begin{aligned}
\int_{\Omega}\left(\nabla^{n} \boldsymbol{u}\right) \cdot\left(\nabla^{n} \boldsymbol{v}\right) \mathrm{d} V & =\int_{\Omega}\left\{\left|\nabla^{n} \boldsymbol{u}\right|^{2}+\alpha^{2}\left|\nabla^{n+1} \boldsymbol{u}\right|^{2}\right\} \mathrm{d} V \\
& =H_{n}+\alpha^{2} H_{n+1} .
\end{aligned}
$$

Thus

$\frac{1}{2} \frac{\mathrm{d}}{\mathrm{d} t}\left(H_{n}+\alpha^{2} H_{n+1}\right)=\int_{\Omega}\left(\nabla^{n} \boldsymbol{u}\right) \cdot\left(\nabla^{n} \boldsymbol{v}_{t}\right) \mathrm{d} V$

which, from (1.4), gives the estimate

$$
\begin{aligned}
\frac{1}{2} \frac{\mathrm{d}}{\mathrm{d} t} & \left(H_{n}+\alpha^{2} H_{n+1}\right)=\int_{\Omega}\left(\nabla^{n} \boldsymbol{u}\right) \\
& \cdot\left\{v \Delta \nabla^{n} \boldsymbol{v}-\nabla^{n}(\boldsymbol{u} \times \operatorname{curl} \boldsymbol{v})\right\} \mathrm{d} V \\
\leq & -v\left(H_{n+1}+\alpha^{2} H_{n+2}\right) \\
+ & \left|\int_{\Omega}\left(\nabla^{n+1} \boldsymbol{u}\right)\left(\nabla^{n-1}(\boldsymbol{u} \times \operatorname{curl} \boldsymbol{v})\right) \mathrm{d} V\right| .
\end{aligned}
$$

Upon separating the two constituent parts of $\boldsymbol{v}=\boldsymbol{u}-\alpha^{2} \Delta \boldsymbol{u}$ within the last term in (4.8), the first is found to satisfy

$$
\begin{aligned}
& \left|\int_{\Omega}\left(\nabla^{n+1} \boldsymbol{u}\right) \cdot\left(\nabla^{n-1}(\boldsymbol{u} \times \operatorname{curl} \boldsymbol{u})\right) \mathrm{d} V\right| \leq\|\boldsymbol{u}\|_{\infty} H_{n+1}^{1 / 2} H_{n}^{1 / 2} \\
& \quad+H_{n+1}^{1 / 2} \sum_{m=1}^{n-1} C_{m}^{n-1}\left\|\nabla^{m} \boldsymbol{u}\right\|_{p}\left\|\nabla^{n-m} \boldsymbol{u}\right\|_{q},
\end{aligned}
$$

where $p, q$ must satisfy $p^{-1}+q^{-1}=1 / 2$ according to Hölder's inequality. The first term on the RHS of (4.10) is the $m=0$ term. Now we use the two Gagliardo-Nirenberg inequalities

$$
\begin{aligned}
& \left\|\nabla^{m} \boldsymbol{u}\right\|_{p} \leq c\left\|\nabla^{n} \boldsymbol{u}\right\|_{2}^{a_{m}}\|\boldsymbol{u}\|_{\infty}^{1-a_{m}} \\
& \left\|\nabla^{n-m} \boldsymbol{u}\right\|_{q} \leq c\left\|\nabla^{n} \boldsymbol{u}\right\|_{2}^{b_{m}}\|\boldsymbol{u}\|_{\infty}^{1-b_{m}}
\end{aligned}
$$

where

$$
\begin{aligned}
& \frac{1}{p}-\frac{m}{d}=a_{m}\left(\frac{1}{2}-\frac{n}{d}\right) \\
& \frac{1}{q}-\frac{n-m}{d}=b_{m}\left(\frac{1}{2}-\frac{n}{d}\right) .
\end{aligned}
$$

Adding the two and noting that $p^{-1}+q^{-1}=\frac{1}{2}$ implies that $a_{m}+b_{m}=1$. In fact this is true in $d$ dimensions. Thus the last term in (4.10) is estimated by

$H_{n+1}^{1 / 2} \sum_{m=1}^{n-1} C_{m}^{n-1}\left\|\nabla^{m} \boldsymbol{u}\right\|_{p}\left\|\nabla^{n-m} \boldsymbol{u}\right\|_{q} \leq c_{n}^{(1)} H_{n}^{1 / 2} H_{n+1}^{1 / 2}\|\boldsymbol{u}\|_{\infty}$

We may approach the second constituent part of $v$ in the same manner as (4.10). After an integration by parts we find

$$
\begin{aligned}
& \left|\int_{\Omega}\left(\nabla^{n+2} \boldsymbol{u}\right) \cdot\left(\nabla^{n-2}\left(\boldsymbol{u} \times \operatorname{curl}\left(-\alpha^{2} \Delta \boldsymbol{u}\right)\right)\right) \mathrm{d} V\right| \\
& \quad \leq c_{n}^{(2)} \alpha^{2} H_{n+1}^{1 / 2} H_{n+2}^{1 / 2}\|\boldsymbol{u}\|_{\infty} .
\end{aligned}
$$


The estimate (4.8) now becomes

$$
\begin{aligned}
\frac{1}{2} \frac{\mathrm{d}}{\mathrm{d} t}\left(H_{n}+\alpha^{2} H_{n+1}\right) \leq & -\frac{1}{2} v\left(H_{n+1}+\alpha^{2} H_{n+2}\right) \\
& +c_{n} v^{-1}\|\boldsymbol{u}\|_{\infty}^{2}\left(H_{n}+\alpha^{2} H_{n+1}\right) \\
& +\left\|\nabla^{n} \boldsymbol{f}\right\|_{2} H_{n}^{1 / 2} \\
& +\alpha^{2}\left\|\nabla^{n+1} \boldsymbol{f}\right\|_{2} H_{n+1}^{1 / 2} .
\end{aligned}
$$

To turn this into an inequality for the $F_{n}$ we add and subtract the negative terms on the right hand side, and break up the last terms to form the group of terms designated as $X_{n}$ :

$$
\begin{aligned}
X_{n}= & \left\|\nabla^{n} \boldsymbol{f}\right\|_{2} H_{n}^{1 / 2}+\alpha^{2}\left\|\nabla^{n+1} \boldsymbol{f}\right\|_{2} H_{n+1}^{1 / 2}+\frac{1}{2} \nu \ell^{-2} \tau^{2}\left\|\nabla^{n} \boldsymbol{f}\right\|_{2}^{2} \\
& +\frac{1}{2} \nu \ell^{-2} \tau^{2} \alpha^{2}\left\|\nabla^{n+1} \boldsymbol{f}\right\|_{2}^{2}
\end{aligned}
$$

with

$$
\begin{gathered}
\frac{1}{2} \frac{\mathrm{d}}{\mathrm{d} t}\left(F_{n}+\alpha^{2} F_{n+1}\right) \leq-\frac{1}{2} v\left(F_{n+1}+\alpha^{2} F_{n+2}\right) \\
+c_{n} v^{-2}\|\boldsymbol{u}\|_{\infty}^{2}\left(F_{n}+\alpha^{2} F_{n+1}\right)+X_{n}
\end{gathered}
$$

where

$$
\begin{aligned}
X_{n} \leq & \left\{\frac{g}{2} H_{n}+\left(\frac{1}{2 g \tau^{2}}+\frac{v}{2 \ell^{2}}\right) \tau^{2}\left\|\nabla^{n} \boldsymbol{f}\right\|_{2}\right\} \\
& +\left\{\frac{g}{2} H_{n+1}+\left(\frac{1}{2 g \tau^{2}}+\frac{v}{2 \ell^{2}}\right) \tau^{2}\left\|\nabla^{n+1} \boldsymbol{f}\right\|_{2}\right\} .
\end{aligned}
$$

To make the coefficients of $H_{n}$ and $\tau^{2}\left\|\nabla^{n} \boldsymbol{f}\right\|_{2}$ equal, choose $g$ to satisfy

$g^{2}-\frac{g v}{\ell^{2}}-\frac{1}{\tau^{2}}=0$.

That is

$2 g=\frac{v}{\ell^{2}}+\left[\left(\frac{v}{\ell^{2}}\right)^{2}+\frac{4}{\tau^{2}}\right]^{1 / 2}$.

Given $\tau$ in (4.2) we have $g \approx \tau^{-1}$ as $G r \rightarrow \infty$. Consequently, $\tau^{-1}=\ell^{2} v^{-1}(G r \ln G r)^{\frac{1}{2}} \leq c \ell^{2} v^{-1} \operatorname{Re}(\ln R e)$

and

$X_{n} \leq \frac{1}{2} \tau^{-1}\left(F_{n}+\alpha^{2} F_{n+1}\right)$.

When applied to (4.17), the previous result yields

$\frac{\mathrm{d} J_{n}}{\mathrm{~d} t} \leq-v J_{n+1}+c_{n}\left(v^{-1}\|\boldsymbol{u}\|_{\infty}^{2}+v \ell^{-2} \operatorname{Re}(\ln R e)\right) J_{n}$

which is (4.4).

The following lemma deals with the $-J_{n+1}$-term in (4.23):

Lemma 1. For $1 \leq p \leq n$, the $J_{n}$ satisfy

$J_{n+1} \geq \frac{1}{2} \frac{J_{n}^{1+\frac{1}{p}}}{J_{n-p}^{1 / p}}$.
Proof. Firstly from (4.1), by writing $F_{n}$ in Fourier transforms one finds

$F_{n}=H_{n}+\tau^{2}\left\|\nabla^{n} \boldsymbol{f}\right\|_{2}^{2}=\int k^{2 n}|\hat{u}|^{2} \mathrm{~d} V_{k}$,

where $|\hat{u}|^{2}$ includes the three components of the fluid velocity and the three components of the forcing. Using Hölder's inequality produces

$$
\begin{aligned}
F_{n} & =\int\left(k^{2(n+q)}|\hat{u}|^{2}\right)^{\frac{p}{p+q}}\left(k^{2(n-p)}|\hat{u}|^{2}\right)^{\frac{q}{p+q}} \mathrm{~d} V_{k} \\
& \leq F_{n+q}^{\frac{p}{p+q}} F_{n-p}^{\frac{p}{p+q}} .
\end{aligned}
$$

Another application of Hölder's inequality also gives the standard result

$N^{-p}\left(\sum_{i=1}^{N} a_{i}\right)^{p+1} \leq \sum_{i=1}^{N}\left|a_{i}\right|^{p+1}$.

Now we find upper and lower bounds for the combination $F_{n}^{p+1}+\alpha^{2 p+2} F_{n+1}^{p+1}$. Inequality (4.26) is used to find an upper bound

$$
\begin{aligned}
F_{n}^{p+1}+\alpha^{2 p+2} F_{n+1}^{p+1} \leq & F_{n+1}^{p} F_{n-p}+\alpha^{2 p+2} F_{n+2}^{p} F_{n+1-p} \\
\leq & \left(F_{n+1}+\alpha^{2} F_{n+2}\right)^{p} \\
& \times\left(F_{n-p}+\alpha^{2} F_{n+1-p}\right) .
\end{aligned}
$$

A lower bound comes from inequality (4.27) with $N=2$

$F_{n}^{p+1}+\left[\alpha^{2} F_{n+1}\right]^{p+1} \geq 2^{-p}\left(F_{n}+\alpha^{2} F_{n+1}\right)^{p+1}$.

Thus we have

$2^{-p} J_{n}^{p+1} \leq J_{n+1}^{p} J_{n-p}$

which gives the result.

The $n=0$ result (4.5) follows from (2.4) by using the same methods.

\section{Length scales}

Now define the quantities $r<n$ (we take $r=n-p$ )

$\kappa_{n, r}(t)=\left(\frac{J_{n}}{J_{r}}\right)^{\frac{1}{2(n-r)}}$,

which act as $t$-dependent wave-numbers and thus have the dimension of inverse length scales. For $r=0$ they are analogous to the $2 n$th moments of the energy spectrum. In what follows we find upper bounds for $\left\langle\kappa_{n, 0}^{2}\right\rangle$. These are bounds on an infinite series of inverse squared length scales.

In Theorem 1 for $r<n$, dividing by the $J_{n}$ and time averaging, we have

$\left\langle\kappa_{1,0}^{2}\right\rangle \leq c_{1} \ell^{-2} R e \ln R e$,

$\left\langle\kappa_{n, r}^{2}\right\rangle \leq c_{n, \alpha} v^{-2}\left\langle\|\boldsymbol{u}\|_{\infty}^{2}\right\rangle+c_{1} \ell^{-2} \operatorname{Re} \ln R e$.

The results for this can be summarized in the following: 
Theorem 2. As $\mathrm{Gr} \rightarrow \infty$, the four time averages are estimated as

$\ell^{2}\left\langle\kappa_{n, 0}^{2}\right\rangle \leq c_{n, \alpha} V_{\alpha}^{\frac{n-1}{n}} R e^{\frac{11}{4}-\frac{7}{4 n}}(\ln R e)^{\frac{1}{n}}+c_{1} R e \ln R e$,

$\ell^{2}\left\langle\kappa_{1,0}^{2}\right\rangle \leq c_{1} R e \ln R e$,

$\ell^{2} v^{-2}\left\langle\|\boldsymbol{u}\|_{\infty}^{2}\right\rangle \leq c V_{\alpha} R e^{11 / 4}$

$\alpha \ell v^{-1}\left\langle\|\nabla \boldsymbol{u}\|_{\infty}\right\rangle \leq c V_{\alpha}^{1 / 4} V_{\ell}^{1 / 2} R e^{35 / 16}$.

Remark. These four results are also listed in Table 1.

Proof. Agmon's inequality says that

$\|\boldsymbol{u}\|_{\infty}^{2} \leq c\left\|\nabla^{2} \boldsymbol{u}\right\|_{2}\|\nabla \boldsymbol{u}\|_{2}=c H_{2}^{1 / 2} H_{1}^{1 / 2}$.

Moreover, we also notice that because $H_{1} \leq H_{2}^{1 / 2} H_{0}^{1 / 2}$ and $U=L^{-3 / 2}\left\langle H_{0}\right\rangle^{1 / 2}$ we have

$\left\langle\|\boldsymbol{u}\|_{\infty}^{2}\right\rangle^{2} \leq c\left\langle H_{0}\right\rangle^{1 / 2}\left\langle H_{2}\right\rangle^{3 / 2} \leq c L^{3 / 2} U\left\langle H_{2}\right\rangle^{3 / 2}$.

which simplifies to ${ }^{5}$

$\ell^{2} v^{-2}\left\langle\|\boldsymbol{u}\|_{\infty}^{2}\right\rangle \leq c V_{\alpha} R e^{11 / 4}$

which is (5.6). With the dimensionless volumes $V_{\alpha}$ and $V_{\ell}$ defined in (1.18), we have

$\ell^{2}\left\langle\kappa_{1,0}^{2}\right\rangle \leq c_{1} R e \ln R e$,

$\ell^{2}\left\langle\kappa_{n, r}^{2}\right\rangle \leq c_{n, \alpha} V_{\alpha} R e^{11 / 4}+c_{1} R e \ln R e$.

For $r=0$ we can improve this by writing

$$
\begin{aligned}
\left\langle\kappa_{n, 0}^{2}\right\rangle & =\left\langle\left(\frac{F_{n}}{F_{1}}\right)^{1 / n}\left(\frac{F_{1}}{F_{0}}\right)^{1 / n}\right\rangle \\
& =\left\langle\left(\kappa_{n, 1}^{2}\right)^{\frac{n-1}{n}}\left(\kappa_{1,0}^{2}\right)^{\frac{1}{n}}\right\rangle \\
& \leq\left\langle\kappa_{n, 1}^{2}\right\rangle^{\frac{n-1}{n}}\left\langle\kappa_{1,0}^{2}\right\rangle^{\frac{1}{n}},
\end{aligned}
$$

and then using the estimates in (5.11) and (5.12) for $n \geq 1$, which gives

$\ell^{2}\left\langle\kappa_{n, 0}^{2}\right\rangle \leq c_{n, \alpha} V_{\alpha}^{\frac{n-1}{n}} R e^{\frac{11}{4}-\frac{7}{4 n}}(\ln R e)^{\frac{1}{n}}+c_{1} \operatorname{Re} \ln R e$.

This is (5.4). Note that when $n=1$ we return to $\ell^{2}\left|\kappa_{n, 0}^{2}\right\rangle \leq$ $c_{1} R e \ln R e$.

It is also possible to estimate $\left\langle\|\nabla \boldsymbol{u}\|_{\infty}\right\rangle$

$$
\begin{aligned}
\left\langle\|\nabla \boldsymbol{u}\|_{\infty}\right\rangle & \leq c\left\langle H_{3}^{1 / 4} H_{2}^{1 / 4}\right\rangle=c\left\langle\kappa_{3,2}^{1 / 2} H_{2}^{1 / 2}\right\rangle \\
& \leq c\left\langle\kappa_{3,2}^{2}\right\rangle^{1 / 4}\left\langle H_{2}\right\rangle^{1 / 2} .
\end{aligned}
$$

\footnotetext{
5 Herein lies the difference between 3D LANS- $\alpha$ equations and the 3D Navier-Stokes equations: in the former we have a bound on $\left\langle H_{2}\right\rangle$ from (2.5) which is missing in the latter.
}

Using (2.5) and (5.12) we find

$$
\alpha \ell v^{-1}\left\langle\|\nabla \boldsymbol{u}\|_{\infty}\right\rangle \leq c\left\langle H_{3}^{1 / 4} H_{2}^{1 / 4}\right\rangle \leq c V_{\alpha}^{1 / 4} V_{\ell}^{1 / 2} R e^{35 / 16},
$$

which is (5.7).

\section{Acknowledgements}

We are enormously grateful to C. Foias and E.S. Titi for many illuminating discussions of the LANS- $\alpha$ equations and their analytical properties. We also thank C.R. Doering for encouraging explanations of the results of [30]. The work of DDH was partially supported by US DOE, Office of Science, Applied Mathematics program of the Mathematical, Information, and Computational Sciences Division (MICS).

\section{Appendix A. Issues concerning the forcing}

\section{A.1. Bounds concerning $\mathrm{Gr}$ and Re}

Doering and Foias [30] split the forcing function $\boldsymbol{f}(\boldsymbol{x})$ into its magnitude $F$ and its "shape" $\boldsymbol{\Phi}$ such that

$\boldsymbol{f}(\boldsymbol{x})=F \boldsymbol{\Phi}\left(\ell^{-1} \boldsymbol{x}\right)$

where $\ell$ is the longest length scale in the force. On the unit torus $\mathbb{I}_{d}, \boldsymbol{\Phi}$ is a mean-zero, divergence-free vector field with the chosen normalization property

$\int_{\mathbb{I}_{d}}\left|\nabla_{y}^{-1} \boldsymbol{\Phi}\right|^{2} \mathrm{~d}^{d} y=1$.

$L^{2}$-norms of $\boldsymbol{f}$ on $\mathbb{I}^{d}$ are

$\left\|\nabla^{N} \boldsymbol{f}\right\|_{2}^{2}=C_{N} \ell^{-2 N} L^{d} F^{2}$

where the coefficients $C_{N}$ refer to the shape of the force but not its magnitude

$C_{M}=\sum_{n}|2 \pi n|^{2 N}\left|\hat{\boldsymbol{\Phi}}_{n}\right|^{2}$.

Doering and Foias [30] showed that various bounds exist such as (among others)

$\left\|\nabla \Delta^{-M} \boldsymbol{f}\right\|_{\infty}=D_{M} F \ell^{2 M-1}$.

The energy dissipation rate $\epsilon$ is

$\epsilon=\left\langle\nu L^{-d} \int_{\Omega}|\nabla \boldsymbol{u}|^{2} \mathrm{~d} V\right\rangle=v L^{-d}\left\langle H_{1}\right\rangle$.

In terms of $F$ the Grashof number in (1.7) becomes

$G r=F \ell^{3} / \nu^{2}$

and the Taylor micro-scale $\lambda_{T}$ is related to $U$ via $\lambda_{T}=$ $\sqrt{\nu U^{2} / \epsilon}$, which is consistent with the definition $\lambda_{T}^{-2}=$ $\left\langle H_{1}\right\rangle /\left\langle H_{0}\right\rangle$.

The LANS- $\alpha$ equations (1.3) and (1.4) can also formally be re-written as

$$
\begin{aligned}
\boldsymbol{u}_{t}+\boldsymbol{u} \cdot \nabla \boldsymbol{u}-v \Delta \boldsymbol{u}+\nabla \tilde{p}= & \left(1-\alpha^{2} \Delta\right)^{-1} \\
& \times\left\{\boldsymbol{f}(\boldsymbol{x})+\alpha^{2} \operatorname{div} \mathbb{T}\right\}
\end{aligned}
$$


where the tensor $\mathbb{T}$ is defined as

$\mathbb{T}=\nabla \boldsymbol{u} \cdot \nabla \boldsymbol{u}+\nabla \boldsymbol{u} \cdot \nabla \boldsymbol{u}^{\mathrm{T}}-\nabla \boldsymbol{u}^{\mathrm{T}} \cdot \nabla \boldsymbol{u}$.

Following the procedure in [30] (pg 296 Eq. 2.9) and multiplying by $\left(-\Delta^{-M}\right) f$ we have

$$
\begin{aligned}
\frac{\mathrm{d}}{\mathrm{d} t} \int_{\mathbb{I}_{d}} \boldsymbol{u} \cdot\left[\left(-\Delta^{-M}\right) \boldsymbol{f}\right] \mathrm{d} V= & -v \int_{\mathbb{I}_{d}} \Delta \boldsymbol{u} \cdot\left[\left(-\Delta^{-M}\right) \boldsymbol{f}\right] \\
& -\int_{\mathbb{I}_{d}} \boldsymbol{u} \cdot \nabla \boldsymbol{u} \cdot\left[\left(-\Delta^{-M}\right) \boldsymbol{f}\right] \mathrm{d} V \\
& +\int_{\mathbb{I}_{d}}\left[\left(-\Delta^{-M}\right) \boldsymbol{f}\right] \cdot\left(1-\alpha^{2} \Delta\right)^{-1} \\
& \times\left\{\boldsymbol{f}+\alpha^{2} \operatorname{div} \mathbb{T}\right\} \mathrm{d} V .
\end{aligned}
$$

Now there are two strategies:

(1) To prove that $G r \leq c R e^{2}$ : integrate all the terms by parts, and take the time average

$$
\begin{aligned}
& \left\langle L^{-d} \int_{\mathbb{I}_{d}} \nabla^{-M} \boldsymbol{f} \cdot\left(1-\alpha^{2} \Delta\right)^{-1} \nabla^{-M} \boldsymbol{f} \mathrm{d} V\right\rangle \\
& \leq\left\langle L^{-d} v \int_{\mathbb{I}_{d}} \boldsymbol{u} \cdot\left[\left(-\Delta^{-M+1}\right) \boldsymbol{f}\right] \mathrm{d} V\right\rangle \\
& \quad-\left\langle L^{-d} \int_{\mathbb{I}_{d}} \boldsymbol{u} \cdot\left[\nabla\left[\left(-\Delta^{-M}\right)\right] \boldsymbol{f}\right] \cdot \boldsymbol{u} \mathrm{d} V\right\rangle \\
& \quad+\left\langle L^{-d} \int_{\mathbb{I}_{d}}\left|\nabla\left[\left(-\Delta^{-M}\right) \boldsymbol{f}\right]\right|\left|\frac{\alpha^{2}}{\left(1-\alpha^{2} \Delta\right)} \mathbb{T}\right| \mathrm{d} V\right\rangle .
\end{aligned}
$$

Using the scaling properties of $\boldsymbol{\Phi}$ and a Fourier transform on the last term

$$
\begin{aligned}
& L^{-d} \int_{\mathbb{I}_{d}}\left|\nabla\left[\left(-\Delta^{-M}\right) \boldsymbol{f}\right]\right|\left|\frac{\alpha^{2}}{\left(1-\alpha^{2} \Delta\right)} \mathbb{T}\right| \mathrm{d} V \\
& \leq D_{M} F \ell^{2 M-1} \int \frac{\alpha^{2} k^{2}}{1+\alpha^{2} k^{2}}|\hat{\boldsymbol{u}}|^{2} \mathrm{~d} V_{k} \\
& \leq D_{M} F \ell^{2 M-1} \int|\hat{\boldsymbol{u}}|^{2} \mathrm{~d} V_{k}
\end{aligned}
$$

where (A.5) defines $D_{M}$. Thus (A.11) turns into

$c_{0} \frac{F^{2} \ell^{2 M}}{1+\alpha^{2} \ell^{-2}} \leq c_{1} v F \ell^{2 M-2} U+c_{2} \ell^{2 M-1} F U^{2}$,

where the $U^{2}$-term contains the contributions from both nonlinear terms and the constants (not explicitly given) contain the shape of the body forcing. Using (A.7) and (A.13) becomes

$G r \leq c\left(R e+R e^{2}\right), \quad G r \rightarrow \infty$,

the only difference from the Navier-Stokes equations being the value of the constant.

(2) To prove that $\epsilon \geq c v^{3} \ell^{-3} L^{-1} G r$ : return to (A.10) and take a different route. Firstly in the Laplacian term use one derivative on $\boldsymbol{u}$ and another on the forcing. Then keep the $\boldsymbol{u} \cdot \nabla \boldsymbol{u}$ advection term. Finally integrate the $\mathbb{T}$-term by parts and exploit the fact that $\left(1-\alpha^{2} \Delta\right)^{-1}$ is a symmetric operator.

$$
\left\langle L^{-d} \int_{\mathbb{I}_{d}}\left(\nabla^{-M} \boldsymbol{f}\right) \cdot \frac{1}{\left(1-\alpha^{2} \Delta\right)} \nabla^{-M} \boldsymbol{f} \mathrm{d} V\right\rangle
$$

$$
\begin{aligned}
\leq & \left\langle L^{-d} v \int_{\mathbb{I}_{d}}|\nabla \boldsymbol{u}| \cdot\left[\left(\nabla \Delta^{-M}\right) \boldsymbol{f}\right]\right\rangle \\
& +\left\langle L^{-d} \int_{\mathbb{I}_{d}}|\boldsymbol{u}||\nabla \boldsymbol{u}|\left|\left(-\Delta^{-M}\right) \boldsymbol{f}\right| \mathrm{d} V\right\rangle \\
& +\left\langle L^{-d} \int_{\mathbb{I}_{d}}\left|\frac{\alpha^{2}}{\left(1-\alpha^{2} \Delta\right)} \nabla\left[\left(-\Delta^{-M}\right) \boldsymbol{f}\right]\right||\mathbb{T}| \mathrm{d} V\right\rangle .
\end{aligned}
$$

Thus (A.15) turns into

$$
\begin{aligned}
c_{0} \frac{F^{2} \ell^{2 M}}{1+\alpha^{2} \ell^{-2}} \leq & c_{1} F v^{1 / 2} \ell^{2 M-1} \epsilon^{1 / 2}+c_{2} F v^{-1 / 2} \ell^{2 M} \epsilon^{1 / 2} U \\
& +c_{3} \alpha^{2} \ell^{2 M-1} F v^{-1} \epsilon .
\end{aligned}
$$

We assume the ordering of length scales as $L \geq \ell \geq \alpha \geq \lambda_{T}$ and use the fact that $U^{2}=\lambda_{T}^{2} \epsilon v^{-1} \leq L^{2} \epsilon \nu^{-1}$. Then (A.16) becomes

$$
\begin{aligned}
\frac{1}{2} c_{0} F & \leq c_{1} v^{1 / 2} \ell^{-1} \epsilon^{1 / 2}+v^{-1} \epsilon\left(c_{2} L+c_{3} \alpha^{2} \ell^{-1}\right) \\
& \leq c_{1} v^{1 / 2} \ell^{-1} \epsilon^{1 / 2}+c_{4} v^{-1} L \epsilon .
\end{aligned}
$$

For $G r \rightarrow \infty$ the last term on the RHS is the dominant one: we have

$\epsilon \geq c_{5} \ell^{-3} L^{-1} v^{3} G r$

which, with a different constant, agrees with the result in Doering and Foias [30]. This inequality is used in the next subsection.

\section{A.2. Forcing and the fluid response}

For technical reasons, we must address the possibility that in their evolution the quantities $H_{n}$ might take small values. Thus we need to circumvent problems that may arise when dividing by these (squared) semi-norms. We follow Doering and Gibbon [31] who introduced the modified quantities in (4.1)

$F_{n}=H_{n}+\tau^{2}\left\|\nabla^{n} \boldsymbol{f}\right\|_{2}^{2}$

where the "timescale" $\tau$ is to be chosen for our convenience. So long as $\tau \neq 0$, the $F_{n}$ are bounded away from zero by the explicit value $\tau^{2} L^{3} \ell^{-2 n} f_{\mathrm{rms}}^{2}$. Moreover, we may choose $\tau$ to depend on the parameters of the problem such that $\left\langle F_{n}\right\rangle \sim\left\langle H_{n}\right\rangle$ as $G r \rightarrow \infty$. To see how to achieve this, let us define

$\tau=\ell^{2} v^{-1}(G r \ln G r)^{-1 / 2}$.

Then the additional term in (A.19) is

$$
\begin{aligned}
\tau^{2}\left\|\nabla^{n} \boldsymbol{f}\right\|_{2}^{2} & =L^{3} v^{-2} \ell^{4-2 n} f_{\mathrm{rms}}^{2}(G r \ln G r)^{-1} \\
& =v^{2} \ell^{-(2 n+2)} L^{3} G r(\ln G r)^{-1}
\end{aligned}
$$

Recalling the a priori bound on the far right hand side of (A.18)

$$
\begin{aligned}
\tau^{2}\left\|\nabla^{n} \boldsymbol{f}\right\|_{2}^{2} & \leq c_{6} \epsilon \ell^{-(2 n-1)} L^{4} v^{-1}(\ln G r)^{-1} \\
& =c_{6}\left(\frac{L}{\ell}\right)^{(2 n-1)} L^{-2(n-1)}\left\langle H_{1}\right\rangle(\ln G r)^{-1} .
\end{aligned}
$$


Using Poincaré's inequality in the form $H_{1} \leq(2 \pi L)^{2(n-1)} H_{n}$, as $G r \rightarrow \infty$ we have

$\frac{\tau^{2}\left\|\nabla^{n} \boldsymbol{f}\right\|_{2}^{2}}{\left\langle H_{n}\right\rangle} \leq c_{6}\left(\frac{L}{\ell}\right)^{(2 n-1)}(\ln G r)^{-1}$.

Hence, the additional forcing term in (A.19) becomes negligible with respect to $\left\langle H_{n}\right\rangle$ as $G r \rightarrow \infty$, so the forcing does not dominate the response.

\section{References}

[1] A.N. Kolmogorov, Dissipation of energy in a locally isotropic turbulence, Dokl. Akad. Nauk SSSR 32 (1941) 141-143 (in Russian); Proc. R. Soc. London A 434 (1991) 15-17 (English translation).

[2] U. Frisch, Turbulence: The Legacy of A.N. Kolmogorov, Cambridge University Press, Cambridge, 1995.

[3] P. Constantin, C. Foias, Navier-Stokes Equations, The University of Chicago Press, Chicago, 1988.

[4] R. Temam, Navier-Stokes Equations and Non-linear Functional Analysis, in: CBMS-NSF Regional Conference Series in Applied Mathematics, SIAM Press, Philadelphia, 1983.

[5] C. Foias, What do the Navier-Stokes equations tell us about turbulence? in: Harmonic Analysis and Nonlinear Differential Equations, in: Contemporary Mathematics, vol. 208, American Mathematical Society Providence, 1997, pp. 151-180.

[6] C. Foias, R. Temam, The connection between the Navier-Stokes Equations Dynamical Systems and Turbulence Theory, in: M.C. Randall, R.H. Rabinowitz, M.E. Turner (Eds.), Directions in Partial Differential Equations, Academic Press, Boston, 1987, pp. 55-73.

[7] M.I. Vishik, A.V. Fursikov, Mathematical Problems of Statistical Hydrodynamics, Kluwer Academic Press, Dordrecht, 1980, pp. 709-737.

[8] J. Leray, Essai sur le mouvement d'un liquide visquex emplissant l'espace, Acta Math. 63 (1934) 193-248.

[9] G. Gallavotti, Some rigorous results about the 3D Navier-Stokes equations, in: R. Benzi, C. Badesvant, S. Ciliberto (Eds.), Les Houches 1992 NATO ASI Meeting on Turbulence in Extended Systems, Nova Science, New York, 1993, pp. 45-81.

[10] B.J. Geurts, D.D. Holm, Regularization modeling for large-eddy simulation, Phys. Fluids 15 (2003) L13-L16.

[11] S. Chen, C. Foias, D.D. Holm, E.J. Olson, E.S. Titi, S. Wynne, The Camassa-Holm equations as a closure model for turbulent channel and pipe flows, Phys. Rev. Lett. 81 (1998) 5338-5341.

[12] D.D. Holm, E.S. Titi, Computational models of Turbulence: The LANS- $\alpha$ model and the role of global analysis, SIAM News 38 (7) (2005).

[13] I. Gjaja, D.D. Holm, Self-consistent wave-mean flow interaction dynamics and its Hamiltonian formulation for a rotating stratified incompressible fluid, Physica D 98 (1996) 343-378.

[14] D.G. Andrews, M.E. McIntyre, An exact theory of nonlinear waves on a Lagrangian-mean flow, J. Fluid Mech. 89 (1978) 609-646.
[15] D.D. Holm, J.E. Marsden, T.S. Ratiu, The Euler-Poincaré equations and semidirect products with applications to continuum theories, Adv. Math. 137 (1998) 1-81.

[16] C. Foias, D.D. Holm, E.S. Titi, The three dimensional viscous CamassaHolm equations and their relation to the Navier-Stokes equations and turbulence theory, J. Dynam. Differential Equations 14 (2002) 1-35.

[17] A. Cheskidov, D.D. Holm, E. Olson, E.S. Titi, On a Leray- $\alpha$ model of turbulence, Proc. R. Soc. A 461 (2005) 629-649. Published online 14 December 2004. doi:10.1098/rspa.2004.1373.

[18] D. Ruelle, Large volume limit of the distribution of characteristic exponents in turbulence, Comm. Math. Phys. 87 (1982) 287-302.

[19] P. Constantin, C. Foias, Global Lyapunov exponents, Kaplan-Yorke formulas and the dimension of attractors for $2 D$-Navier-Stokes equations, Comm. Pure Appl. Math. 38 (1985) 1-27.

[20] P. Constantin, C. Foias, R. Temam, Attractors representing turbulent flows, Mem. Amer. Math. Soc. 53 (314) (1985).

[21] P. Constantin, C. Foias, R. Temam, On the dimension of attractors in twodimensional turbulence, Physica D 30 (1988) 284-296.

[22] P. Constantin, Collective $L^{\infty}$-estimates for families of functions possessing orthonormal derivatives, Indiana Univ. Math. J. 36 (1987) 603-615.

[23] R. Temam, Infinite Dimensional Dynamical Systems in Mechanics and Physics, in: Applied Mathematical Sciences Series, vol. 68, SpringerVerlag, New York, 1988.

[24] E. Lieb, W. Thirring, Inequalities for the moments of the eigenvalues of the Schrödinger equation and their relation to Sobolev spaces, in: E. Lieb, B. Simon, A. Wightman (Eds.), Studies in Mathematical Physics: Essays in honour of Valentin Bargman, Princeton Univ. Press, Princeton, NJ, 1976.

[25] O.A. Ladyzhenskaya, The Mathematical Theory of Viscous Incompressible Flow, Gordon and Breach, New York, 1963.

[26] J. Serrin, in: R.E. Langer (Ed.), The Initial Value Problem for the Navier-Stokes Equations, Nonlinear Problems, University of Wisconsin Press, Madison, 1963.

[27] C. Foias, Statistical study of Navier-Stokes equations I, Rend. Sem. Mat. Univ. Padova 48 (1972) 219-348; Rend. Sem. Mat. Univ. Padova 49 (1973) 9-123.

[28] C. Foias, G. Prodi, Sur les solutions statistique des équations de Navier-Stokes, Ann. Mat. Pura Appl. 4 (111) (1976) 307-330.

[29] C.R. Doering, J.D. Gibbon, Applied Analysis of the Navier-Stokes Equations, Cambridge University Press, Cambridge, 1995.

[30] C.R. Doering, C. Foias, Energy dissipation in body-forced turbulence, J. Fluid Mech. 467 (2002) 289-306.

[31] C.R. Doering, J.D. Gibbon, Bounds on moments of the energy spectrum for weak solutions of the three-dimensional Navier-Stokes equations, Physica D 165 (2002) 163-175.

[32] J.D. Gibbon, C.R. Doering, Intermittency \& regularity issues in threedimensional Navier-Stokes turbulence, Arch. Ration. Mech. Anal. 177 (2005) 115-150.

[33] C. Foias, D. Holm, E.S. Titi, The Navier-Stokes-alpha model of fluid turbulence, Physica D 152 (2001) 505-519. 